Mit dem nachfolgenden Kurzbeitrag möchte Ernst Zeller, Titularprofessor an der Rechtswissenschaftlichen Fakultät der Universität Zürich, auf die Problematik derjenigen Arbeitsunfähigkeitszeugnisse aufmerksam machen, die einer «kritischen Überprüfung» nicht standhalten. Gemäss den von ihm angeführten medizinischen Referenzen trifft diese Einstufung in bis zu $75 \%$ der Fälle zu. Der Autor hat zu diesem Thema eine Studie verfasst, die er der Leserschaft als Beilage - und somit ausserhalb des redaktionellen Zuständigkeitsbereichs - zu dieser Ausgabe der Schweizerischen Ärztezeitung (SÄZ) vorlegt. Im Sinne der Funktion der SÄZ als offene Diskussionsplattform für gesundheitspolitische Themen publizieren wir seine zusammenfassenden Aussagen gemeinsam mit zwei Kommentaren. Wir danken Dieter Kissling, ärztlicher Leiter des Instituts für Arbeitsmedizin in Baden, und Hanspeter Kuhn, Leiter des Rechtsdienstes der FMH, für die Bereitschaft, eine einordnende Kommentierung aus juristischer und (arbeits-)medizinischer Sicht vorzunehmen.

Die Redaktion

\title{
Krankheit und Arbeitsunfähigkeit - eine interdisziplinär abgestützte Studie zu einem drängenden Thema
}

\section{Ernst Zeller*}

Der Autor dankt lic. iur. Philip R. Bornhauser für seine Mitarbeit.

1 Linden M, Weidner C. Arbeitsunfähigkeit bei psychischen Störungen. Der Nervenarzt. 2005;76(11):1421-31.

2 www.rwi.uzh.ch/tit-zeller/publ kationen/krankheitundarbeits unfaehigkeitzuerich2007.pdf

Korrespondenz:

Prof. Dr. iur. Ernst Zeller

Postfach 1066

CH-8021 Zürich
Das Bundesamt für Statistik nennt für das Jahr 2005 in der Schweiz krankheitsbedingte Absenzen von 203 Millionen Stunden und einen dadurch verursachten Anteil von $77 \%$ am gesamten jährlichen Absenzenvolumen. Das ergibt umgerechnet statistisch gut 53 Stunden pro Vollzeitarbeitsstelle und Jahr.

Die medizinische Fachliteratur in der Schweiz und in Deutschland weist eine hohe Zahl von unwahren ärztlichen Arbeitsunfähigkeitsattesten bzw. von reinen Gefälligkeitszeugnissen nach. Die Ärztegesellschaft des Kantons Zürich benannte die Erscheinung im Jahr 2000 als «Absentismus». Eine medizinische Studie über Arbeitsunfähigkeit wegen psychischer Störungen kommt zum Schluss: «Arbeitsunfähigkeits-Feststellungen durch Ärzte halten in etwa $75 \%$ der Fälle einer kritischen Überprüfung nicht stand» (vgl. dazu Linden/Weidner, Forschungsgruppe Psychosomatische Rehabilitation an der Charité und der Rehabilitationsklinik Seehof, Teltow/Berlin [1]).

Die Aussagen der medizinischen Studien sind klar und gelten unabhängig von länderspezifischen Regelungen, weil diagnostische Aussagen medizinisch aufgrund internationaler Standards zu machen sind.

Fernbleiben von der Arbeit unter unwahrem Vorschützen von Arbeitsunfähigkeit wegen Krankheit mit Hilfe von leichtfertig ausgestellten, unwahren Arztattesten ist somit ein gesellschaftliches Problem, das die Volkswirtschaft und vor allem die ehrlichen und redliche Arbeitnehmenden schädigt und diese für ihre Rechtschaffenheit bestraft.

Mit Arbeitsunfähigkeitsattesten geben Ärzte, oft die Hausärzte Fachbeurteilungen ab. Die rechtlichen Aspekte regeln Gesetz und Rechtsprechung. Auch die Ärzte sind an das Recht gebunden, und das Recht ist auf korrekte Arbeit der Ärzteschaft angewiesen. Somit sind die medizinischen und die rechtlichen Aspekte rund um die Ausstellung von ärztlichen Arbeitsunfähigkeitsbescheinigungen für die Ärzteschaft von hoher Relevanz.
Als Beilage zu dieser Nummer der Schweizerischen Ärztezeitung erscheint dazu ein Aufsatz des Schreibenden. Der Aufsatz ist inhaltlich interdisziplinär. Er dokumentiert medizinische und rechtliche Befunde und ist im Internet abrufbar [2].

Das aus der Medizin stammende Erfordernis, Gesundheitsbeeinträchtigung einerseits und deren Grad, der Krankheitswert hat, anderseits zu unterscheiden, wird in der ärztlichen Praxis allzuoft vernachlässigt. Dasselbe gilt für den Umgang mit der Fragestellung, ob bei Vorliegen von Krankheitswert eine Arbeitsaussetzung nötig ist. Der Pflicht zu Objektivierung gegenüber subjektiven Patientenschilderungen wird oft nicht nachgelebt. Einer Behauptung über Mobbing z.B. einfach zu glauben, ohne die Abklärungsschritte laut den beruflichen Pflichten durchzuführen, degradiert den Arzt zum Zuhörer von Geschichten.

Der von den Medizinern und von deren Wissenschaft festgestellte Zustand ist unerfreulich und nicht angenehm. Er wird deshalb in der erwähnten Studie sachlich und dokumentarisch geschildert.

Entgegen einer öfters anzutreffenden Meinung gehört es nicht zu den ärztlichen Pflichten, Arbeitnehmende ohne medizinische Indikation, aber mit Blick auf die allgemeine Wirtschaftslage zu deren Schutz krank zu schreiben. Entweder ist jemand krank, und die Krankheit hat die Folge von Arbeitsunfähigkeit, oder dem ist nicht so. Gesellschaftspolitik über Krankschreibungen hat die Ärzteschaft nicht zu machen. Aufgegeben ist dem Arzt gewiss der Einsatz für die Gesundheit der ihm anvertrauten Menschen.

Teilweise hat die Justiz erkannt, dass das Problem der Parteilichkeit zufolge Kundschaftsnähe beim Hausarzt besteht. Vertrauensärzte sind solchen Risiken weniger ausgesetzt (weil vom Arbeitgeber und vom Patienten unabhängiger). Ein Fachausweis, Arbeitsunfähigkeit attestieren zu dürfen, könnte den Hausarzt zu diesem Thema vielleicht von der Last des Spannungsfelds zur Patientennähe befreien. 
Wenn Mitarbeitende der Arbeit unter unwahrem Vorschützen von Arbeitsunfähigkeit wegen Krankheit fernbleiben, sind die redlichen Arbeitnehmerinnen und Arbeitnehmer die Bestraften. Die Redlichen leisten die Zusatzarbeit, die Redlichen vor allem zahlen die - zu Lasten der Solidargemein- schaft - steigenden Prämien für Krankentaggeldversicherungen.

Redliche, pflichtbewusste Ärzte sind sich dieser sich verschärfenden Lage bewusst und stellen keine Arbeitsunfähigkeitsatteste mit leichter Hand aus.

\section{Kommentar aus arbeitsmedizinischer Sicht}

Dieter Kissling

Korrespondenz:

Dr. med. Dieter Kissling

ifa Institut für Arbeitsmedizin

Kreuzweg 3

CH-5400 Baden

Tel. 0562054444

Fax 0562057616

dieter.kissling@arbeitsmedizin.ch

www.arbeitsmedizin.ch
Herr Professor Zeller fordert in seiner Studie zu Recht Sachlichkeit zum Thema Arztzeugnis, das von Arbeitgeberschaft, Arbeitnehmerschaft, Justiz und Ärzteschaft stark emotional diskutiert wird. Im Gegensatz zu seiner Forderung nach Sachlichkeit schreibt er anklagend und polarisierend, was nicht dazu beitragen wird, dass sich die breite Ärzteschaft dem Thema verantwortungsvoll und wohlwollend zuwenden wird.

Es ist dringend notwendig, dass wir Ärzte uns mit dem Thema Arztzeugnis auseinandersetzen und wir uns der verantwortungsvollen Aufgabe, die uns von Gesellschaft und Staat übertragen wurde, medizinisch und juristisch korrekt annehmen, jedoch dabei nie vergessen, unseren Patienten gerecht zu werden.

Der Vorwurf von leichtfertig ausgestellten unwahren Arztattesten bis hin zu Gefälligkeitszeugnissen gilt sicher für Einzelfälle, darf aber nicht im Giesskannenprinzip über die Ärzteschaft gegossen werden. Der Vorwurf der Subjektivität ist haltbar, und wir Ärzte sind uns dessen wohl bewusst. Das Bestreben der Ärzte um eine Objektivierung dürfte in den meisten Fällen stattfinden. Die Ausstellung des Arztzeugnisses ist jedoch nicht ein Gerichtsprozess mit einem Urteil, in dem eine Anhörung aller Beteiligten erfolgt. Viele Krankheitsbilder sind weit davon entfernt, objektiviert zu werden. Burn-out wird man nicht mit EKG, CT und Fiebermesser objektiv feststellen können.

Ein Arztzeugnis bleibt letztlich immer eine Parteibehauptung, die aber seit Jahrzehnten Bestand hat und akzeptiert ist. Dies wohl sicher auch darum, weil Arztzeugnisse in den meisten Fällen objektiv und nachvollziehbar sind. Als Arbeitsmediziner werde ich von Arbeitgebern oft um eine Überprüfung der Arztzeugnisse angefragt. In den meisten Fällen sind die Entscheide der Kollegen nachvollziehbar und korrekt. Die häufigste Schwachstelle, die ich im Alltag erkennen muss, ist die Unkenntnis des behandelnden Arztes über die Bedingungen und Anforderungen am Arbeitsplatz. Hier wünsche ich mir einen stärkeren Dialog zwischen Ärzteschaft und Arbeitgebern. Der Dialog würde die Schaffung von temporären leichteren Einsatzmöglichkeiten durch die Arbeitgeber ermöglichen und dem Arzt Grundlage für eine höhere Objektivität geben.

Arbeitgeberseitig ist die Einführung einer nachhaltigen betrieblichen Gesundheitsförderung das Präventionsprogramm gegen Absenzen. Motivierte und am Arbeitsplatz wohlbefindliche Mitarbeitende werden nicht blaumachen. Wir wissen, dass letztendlich die Hälfte aller Kurzabsenzen motivational bedingt ist und die Arbeitgeber sich Gedanken machen müssen, wie sie diesem Missstand begegnen sollen. Es kann nicht Aufgabe des Arztes sein, Arbeitnehmende, die wegen misslicher Verhältnisse am Arbeitsplatz ihre Krankheit unbewusst aggravieren, zu motivieren. Motivational bedingte Arbeitsunfähigkeiten sind weit vom Blaumachen entfernt, da Blaumachen ein bewusster Prozess ist. Als Arbeitsmediziner begegne ich oft Situationen, in denen ich den Patienten wegen der Gefahr einer Verschlechterung der Gesundheit wegen der Arbeitsplatzverhältnisse präventiv aus dem Verkehr ziehen muss, um ihn noch in einem Gesundheitszustand zu erhalten, in dem er arbeitsmarktfähig bleibt. Dies ist nicht Unterstützung eines Blaumachers, sondern aktive Prävention. Als typisch dafür kann der Burn-out-Prozess erwähnt werden. Die geringe Sensibilität für eigene Gefühle und Bedürfnisse dieser Patienten führt dazu, dass sie gar nicht bemerken, wie schlecht es ihnen geht. Hier habe ich die Aufgabe und Pflicht, diese Patienten sogar gegen den eigenen Willen arbeitsunfähig zu schreiben.

Tatsächlich ist es jedoch so, dass wir zum Wohle des Patienten oft Überlegungen in der Beurteilung der Arbeitsunfähigkeit einbeziehen, die nach der heutigen Rechtsprechung keinen Platz haben dürfen. Insbesondere dürfen Gedanken über den aktuellen Arbeitsmarkt oder soziokulturelle Überlegungen nicht in unsere Entscheidung über die Arbeitsunfähigkeit einfliessen. Entscheidend kann allein sein, ob der Arbeitnehmende momentan für seine konkrete Arbeit aus gesundheitlichen Gründen ganz oder teilweise arbeitsunfähig ist.

Sicher entspricht es auch der Tatsache, dass die Verweigerung eines Arztzeugnisses zu ellenlangen Diskussionen und Erklärungen mit dem Patienten und seinem sozialen Umfeld führt, die mühsam 
sind und sehr viel Zeit und Energie in Anspruch nehmen. Vor diesem Hintergrund ist die Ausstellung einer Arbeitsunfähigkeit mit viel weniger Aufwand verbunden. Ja, wir erhalten sogar Anerkennung dafür von seiten des Patienten.

Sicher stellen wir oft Zeugnisse aus, wenn Patienten Befindlichkeitsstörungen haben. Nehmen wir das Beispiel einer Arbeitsunfähigkeit im Kündigungsfall. Würde ein Gekündigter nicht über eine Krankentaggeldversicherung verfügen, würde er auch im Kündigungsfall aus existentiellen Gründen weiter zur Arbeit gehen müssen, wäre also arbeitsfähig. Es kann und darf nicht sein, dass die Existenz einer Versicherung darüber entscheidet, ob ein Patient arbeitsfähig oder nicht arbeitsfähig ist.

Sicher besteht eine Einstellungsänderung gegenüber der Arbeitsfähigkeit chronischer Schmerzpatienten. Die frühere Überzeugung, Schmerzpatienten könnten nicht mehr arbeiten, ist der Meinung gewichen, dass diese sehr wohl arbeiten können, wenn ihr Gesundheitszustand nicht durch die Arbeit verschlechtert wird.

Herr Professor Zeller wirft uns Parteilichkeit infolge Kundschaftsnähe und einfacher finanzieller Überlegungen vor. Ja, wir sind Partei, und auch wir sind gezwungen, uns tagtäglich finanzielle Überlegungen zu machen. Doch wir streben nach Objektivität. Aber auch Vertrauensärzte sind Partei und somit ebensowenig oder ebensogut geeignet für die Beurteilung der Arbeitsfähigkeit. Die Forderung nach einem Fachausweis erscheint mir hypertroph.

Das Thema der Arztzeugnisse ist hochkomplex, nicht nur aus juristischer, sondern auch aus psychodynamischer Sicht unserer Patienten. Somit ist das Positive am Artikel von Herrn Professor Zeller, dass ein Thema, das die Gemüter aller Involvierter seit Jahren bewegt, auf den Tisch kommt.

Anstatt einen Fachausweis zu verlangen, müssen die Überlegungen der juristischen, medizinischen und psychodynamischen Dimensionen des Arztzeugnisses in die Weiterbildung der Ärzte einfliessen. Klare Richtlinien müssen erstellt und verbreitet werden. Wer wäre besser dazu geeignet als die FMH? Dass diese Thematik interdisziplinär aufgearbeitet werden muss, steht ausser Zweifel. Es wäre wünschenswert, Herr Professor Zeller würde sich daran beteiligen, dies jedoch in einer wohlwollenden, unterstützenden Geisteshaltung.

\section{Kommentar aus Sicht des FMH-Juristen}

\section{Hanspeter Kuhn}

* Die Stressstudie des SECO hält fest: «Wir schätzen die jährlichen Kosten von Stress an schweizerischen Arbeitsplätzen auf mindestens 4 Milliarden Franken» [1].

** Aktuellstes Beispiel: die 2007 partnerschaftlich eingeführten differenzierten Arbeitsunfähigkeitszeugnisse im Kanton St. Gallen: «Einfaches Arztzeugnis»; «Arbeitsplatzbeschreibung» (vom Arbeitgeber erstellt) sowie «Detailliertes Arztzeugnis» (basierend auf Arbeitsplatzbeschreibung).

1 Kiener A, Graf M, Fahrni M. Die Kosten des Stresses in der Schweiz. Bern: SECO; 2003.

Korrespondenz:

Hanspeter Kuhn

FMH

Postfach 170

CH-3000 Bern 15

Tel. 0313591111

Fax 0313591112

lex@fmh.ch
Der Beitrag und die Beilage von Prof. Zeller haben den Vorzug, eine wichtige Thematik in Erinnerung zu rufen; in der Schweizerischen Ärztezeitung sollen auch kontroverse Standpunkte vertreten werden können. Versuch einer Einordnung aus meiner Sicht: Gemäss dem Beitrag von Prof. Zeller müsste auch in der Schweiz der Anteil von Gefälligkeitszeugnissen hoch sein. Ich stelle fest, dass ein erfahrener Arbeitsmediziner wie Dieter Kissling die reale Lage anders beurteilt.

Der Titel von Beitrag und Beilage nimmt in Anspruch, Krankheit und Arbeitsunfähigkeit abzuhandeln. Der Inhalt diskutiert dann ausschliesslich die - wichtige - Thematik von Gefälligkeitszeugnissen. Dass die Belastungen am Arbeitsplatz zuweilen so hoch sind, dass die Ärztin einen Arbeitnehmer zu seinem eigenen Schutz und gegen seinen Willen krank schreiben muss, erfahren wir vom Arbeitsmediziner*; solches passt nicht in das durch den Beitrag von Prof. Zeller vermittelte Weltbild.

Der Beitrag und die Beilage von Professor Zeller setzen sich vehement für routinemässig wesentlich gründlichere - und damit wesentlich teurere - Untersuchungen der Arbeitsunfähigkeitssituation ein. Ich stelle fest: Wo sich kantonale Ärztegesellschaft und kantonaler Arbeitgeberverband an den Tisch setzen und gemeinsame Lösungen** erarbeiten, wird aus Kosteneffizienzgründen differenziert: In den vielen klaren und unkomplizierten Fällen soll der Arzt keinen zusätzlichen unnötigen Abklärungsaufwand betreiben und auch nur ein kurz gehaltenes Zeugnis ausstellen. Nur in den deutlich selteneren Fällen, in denen die Situation nicht für alle klar erscheint, soll gründlicher abgeklärt und ausführlicher berichtet werden - dann lohnen sich auch die hohen Kosten dafür.

Ärzte und auch Arbeitgeber können die heute schwierige Kommunikation verbessern. Ein wirksamer Beitrag des Arbeitgebers besteht darin, dass er eine arbeitsmedizinisch beratende Ärztin (oder einen solchen Arzt) beauftragt, die möglichst das Vertrauen aller geniesst: des Arbeitgebers, der Arbeitnehmer und der behandelnden Ärzte. Sie kann den behandelnden Ärzten unkompliziert und zuverlässig über die gesundheitlichen Anforderungen der einzelnen Arbeitsplätze Auskunft geben; sie dient als Frühwarnsystem für den Arbeitgeber (Burn-outProphylaxe) und senkt durch ihre Schnittstellenfunktion die Wahrscheinlichkeit von Gefälligkeitszeugnissen (genauso wie ein allseitig anerkannter Vertrauensarzt einer Krankenkasse bekanntlich wirksam generalpräventiv wirkt). 